\title{
Contrarreforma psiquiátrica brasileira e medicalização do sofrimento mental na pandemia de Covid-19*
}

Brazilian psychiatric counter-reformation and medicalization of mental distress in the Covid-19 pandemic

\author{
Marcos Roberto Vieira Garcia** \\ Simone Cristina de Amorim*** \\ Gelberton Vieira Rodrigues**** \\ Luiz Henrique Franco Mendonça*****
}

\begin{abstract}
Resumo - O presente artigo buscou compreender os caminhos pelos quais os agentes da contrarreforma psiquiátrica no Brasil se utilizaram da pandemia como forma de expandir sua influência no debate público e em políticas governamentais, tendo como pano de fundo a medicalização do sofrimento mental associado aos efeitos da pandemia da Covid-19. A análise de conteúdo de portarias recentes, de publicações em redes sociais e de entrevistas de atores ligados à Associação Brasileira de Psiquiatria e ao Ministério da Saúde evidenciou a medicalização do sofrimento mental por meio da construção discursiva de uma suposta "quarta onda" da Covid-19, que teria como efeito uma epidemia de transtornos mentais. A resposta do poder público federal referendou a estratégia medicalizante, por meio da liberação suplementar de 650 milhões de reais para compra de psicotrópicos e da disseminação de material produzido pela indústria farmacêutica pelo Ministério da Saúde.
\end{abstract}

Palavras-chave: medicalização; sofrimento mental; Covid-19; reforma psiquiátrica.

\begin{abstract}
This article aims to understand the ways in which psychiatric counter-reformation agents in Brazil used the pandemic as a way to expand their influence in public debate and government policies, against the background of the medicalization of mental distress associated with the effects of the Covid-19 pandemic. The content analysis of recent government ordinances, publications on social networks and interviews with players linked to the Brazilian Association
\end{abstract}

\footnotetext{
* Para este artigo, Amorim reconhece o apoio parcial da Fundação de Amparo à Pesquisa do Estado de São Paulo (FAPESP), através da Bolsa de Treinamento Técnico III processo no 2019/19524-6, vinculada ao projeto temático "Vulnerabilidades de jovens às IST/HIV e à violência entre parceiros: avaliação de intervenções psicossociais baseadas nos direitos humanos" processo no 2017/25950-2.

** Doutor em Psicologia Social (USP), Professor Associado (UFScar).E-mail: mgarcia@ufscar.br. ORCID: http://orcid.org/ 0000-0002-5668-2923.

*** Doutoranda em Educação (UFScar), Mestre em Filosofia (Unicamp).E-mail: sim.psicologa@gmail.com. ORCID: https://orcid.org/0000-0002-0897-6484.

**** Doutorando em Educação (UFScar); Mestre em Educação Sexual (Unesp). E-mail: gelbertonrodrigues@gmail.com. ORCID: https://orcid.org/0000-0001-5975-1843.

***** Mestrando em Estudos da Condição Humana (UFSCar). E-mail: henrique_91fm@yahoo.com.br. ORCID: https:/ /orcid.org/0000-0001-9810-8797.
} 
\} CONTRARREFORMA PSIQUIÁTRICA BRASILEIRA - GARCIA, M. R. V.; AMORIM, S. C.; RODRIGUES, G. V.; MENDONÇA, L. H. F. \} DOI: $10.12957 /$ REP.2022.63525

\begin{abstract}
of Psychiatry and the Ministry of Health showed the medicalization of mental distress through the discursive construction of a supposed "fourth wave" of Covid-19, which would have as effect an epidemic of mental disorders. The response of the federal government endorsed the medicalizing strategy, through the additional release of 650 million reais for the acquisition of psychotropic medicines and the dissemination by the Ministry of Health of material produced by the pharmaceutical industry.
\end{abstract}

Keywords: medicalization; mental distress; Covid-19; psychiatric reform.

O presente artigo aborda a medicalização no campo da saúde mental durante a pandemia da Covid-19 no cenário brasileiro, a partir da análise de conteúdo de portarias recentes, de publicações em redes sociais e de entrevistas e lives de atores sociais ligados à Associação Brasileira de Psiquiatria (ABP) e ao Ministério da Saúde. A pesquisa realizada busca compreender os caminhos pelos quais os agentes da contrarreforma psiquiátrica no Brasil se utilizaram da pandemia como meio de expandir sua influência no debate público e em políticas governamentais, tendo como pano de fundo a medicalização do sofrimento mental associado aos efeitos da pandemia.

A psiquiatria clássica se desenvolveu intrinsecamente a partir de estratégias medicalizantes. Tendo como função a higiene do corpo social, sua institucionalização no Ocidente demandou a patologização das supostas anormalidades sob o signo de doenças mentais, garantindo ao poder psiquiátrico a supervisão das estratégias de controle da sociedade, a partir do século XIX (FOUCAULT, 2010). No último quarto do século XX, com o DSM III, a medicalização no campo psiquiátrico teve um novo influxo, dada a necessidade de identificação de cada suposto transtorno por critérios acessíveis, o que levou a uma superespecificação dos diagnósticos e ao aumento exponencial das categorias listadas (RUSSO, 2004). Movimentos de resistência à lógica medicalizante no campo da saúde mental, porém, têm se desenvolvido há algumas décadas, denunciado os mecanismos de controle e interesse financeiro subjacentes (AMARANTE; NUNES, 2018).

Desde seu início, a pandemia da Covid-19 tem suscitado debates no cenário mundial em relação a suas consequências na esfera da saúde mental. Para autores próximos à psiquiatria de tradição biomédica, uma suposta "epidemia de distúrbios mentais" acompanharia a propagação da Covid-19, tanto por questões econômicas, como pelos próprios efeitos do distanciamento social e dos lutos pela perda de pessoas próximas. O contraponto ao viés medicalizante dessa abordagem do sofrimento mental, porém, se deu desde o início desse debate. Para Morgan e Rose (2020), ansiedade, tristeza ou dificuldade para dormir são respostas compreensíveis às mudanças sociais advindas com a pandemia, devendo ser entendidas como uma forma de sofrimento social. Ao entendê-las como sintomas de doenças mentais, corre-se o risco de patologizar processos de adaptação comuns a um período de incertezas. 
Mais recentemente, uma série de pesquisas tem mostrado que as previsões da presumida "epidemia de distúrbios mentais", mesmo aquelas que utilizam referenciais da psiquiatria de tradição biomédica, não se confirmaram. No Reino Unido, Fancourt, Steptoe e Bu (2021) acompanharam, em três momentos, 36.520 pessoas entre março e agosto de 2020, com escalas de mensuração de depressão e ansiedade. Os níveis mais elevados de depressão e ansiedade ocorreram nos estágios iniciais de lockdown, mas diminuíram rapidamente a níveis pré-pandêmicos. Uma análise de três coortes na Holanda mostrou uma ligeira diminuição, durante a pandemia, nos sintomas de pessoas diagnosticadas com transtornos depressivos, ansiosos ou obsessivo-compulsivos (PAN et al., 2021). Em pesquisa brasileira com 2.117 participantes do Estudo Longitudinal de Saúde do Adulto (Elsa Brasil), com idade média de 62,3 anos, os resultados das escalas de mensuração dos chamados "transtornos mentais comuns", aplicadas durante a pandemia, foram similares aos obtidos antes de seu advento. Houve uma diminuição estatisticamente significativa, contudo, ao longo de três avaliações após o início da pandemia, nos escores para depressão e sintomas de estresse (BRUNONI et al., 2021).

Apesar das evidências que apontam no sentido contrário, no cenário brasileiro o processo de medicalização do sofrimento mental como consequência da pandemia da Covid-19 tem sido bastante eloquente e orientador de políticas públicas pautadas neste processo.

\section{A contrarreforma psiquiátrica brasileira e seus tentáculos}

No cenário brasileiro, podemos identificar 2015 como um ano marcado por disputas que favoreceram o fortalecimento da contrarreforma psiquiátrica no Brasil e, com ela, a expansão da lógica medicalizante. Publicações como as de Duarte et al. (2020), Garcia (2019), Lobosque (2018) e Passos (2018) permitem detectar que, a partir de 2015, as políticas públicas de saúde mental, com garantias às conquistas historicamente recentes do cuidado integral e em liberdade, foram sendo dilapidadas por grupos com posicionamentos corporativistas e conservadores, sejam eles religiosos, sejam eles categorizadores dos padrões dos sinais e sintomas. Dentre estes, a ABP se mantém como a principal agente da contrarreforma psiquiátrica brasileira até o presente.

Passos (2018) aponta que, especialmente ao final de 2015, na tentativa de frear o impeachment de Dilma Roussef, foi feita uma "dança das cadeiras" nos ministérios. Com a mudança do ministro da saúde houve, também, a mudança na coordenação de Saúde Mental, Álcool e outras Drogas. A gestão que assumiu deixou de estar posicionada em conformidade com a reforma psiquiátrica e a luta antimanicomial, se afastando, portanto, dos princípios e diretrizes do Sistema Único de Saúde (SUS). Não por acaso, 
Garcia (2019) aponta também que, desde 2015, a campanha "Setembro Amarelo", que se apresenta como campanha de prevenção ao suicídio, ganhou visibilidade nacional, em uma iniciativa que uniu Centro de Valorização da Vida (CVV), Conselho Federal de Medicina (CFM) e Associação Brasileira de Psiquiatria (ABP); e que atrelou o suicídio unicamente ao ato em decorrência de doenças e transtornos mentais, para o qual a prevenção com "tratamento precoce" tem viés nitidamente medicalizante.

No período de 2016 a 2018, o que antes se insinuara foi acentuado. Em 2016-2017 se iniciaram os ataques às políticas de saúde mental (PASSOS, 2018). Indicado pelas forças conservadoras da psiquiatria nacional, Quirino Cordeiro Jr. assumiu o cargo no início de 2017 e os ataques à política de saúde mental passaram a ser diretos, com declarações públicas da gestão federal para favorecer a expansão dos leitos psiquiátricos, corroboradas por emissão de sucessivas portarias. Em fevereiro de 2019, as nomeações de Cordeiro Jr. como Secretário Nacional de Cuidados e Prevenção às Drogas do Ministério da Cidadania, e de Maria Dilma Teodoro para a coordenação de Saúde Mental, Álcool e outras Drogas do Ministério da Saúde - seguida por sua substituição, em fevereiro de 2021, por Rafael Bernardoni Ribeiro -, também alinhados com as forças conservadoras da psiquiatria, evidenciam a expansão da influência crescente da ABP sobre as políticas públicas no campo da saúde mental. Para Lobosque (2018), a principal reivindicação da $\mathrm{ABP}$ é a da reabertura dos hospitais psiquiátricos, se aliando, também, aos que promovem o enclausuramento das pessoas que usam álcool e outras drogas nas chamadas comunidades terapêuticas; o contrário, portanto, das práticas de cuidado em liberdade e das conquistas da reforma psiquiátrica.

Diante da eclosão da pandemia de Covid-19, a ABP divulgou em seu site um ofício endereçado ao então ministro da saúde Luiz Henrique Mandetta, em que solicita a inclusão de psicotrópicos na Relação Nacional de Medicamentos Essenciais (Rename) ${ }^{1}$, e "alertou" sobre risco de descontinuidade de alguns medicamentos ao Ministério da Saúde, sob a justificativa do uso destes para prevenir suicídios, internações e cronificação de "doenças mentais", e no intuito de diminuir custos do sistema de saúde e assistência social (ASSOCIAÇÃO BRASILEIRA DE PSIQUIATRIA, 2020). A ABP sugeriu, inclusive, dispensação dos medicamentos em farmácia popular e produção em laboratórios farmacêuticos vinculados ao Ministério da Saúde e ao Governo Federal dos psicofármacos que "perderam a patente", com produção descontinuada pela indústria, evidenciando a centralidade da medicação na concepção da psiquiatria biomédica que sustenta. Além disso, ignorou as interações entre o vírus e sua expansão desigual nos segmentos étnicoraciais e de classes mais expostos. Com o recrudescimento da pandemia de

1'A Relação Nacional de Medicamentos Essenciais (Rename) traz uma lista, previamente aprovada pelo Ministério da Saúde, para dispensação medicamentosa no SUS. Disponível em: http://conitec.gov.br/images/Rename-2020-final.pdf. Acesso em: 24 jun. 2021. 
Covid-19 no Brasil, a ABP passou a defender publicamente a ideia de que teríamos uma "epidemia de distúrbios mentais" associada à Covid-19, a partir do alarde em torno de uma suposta "quarta onda" da pandemia.

A origem do termo "quarta onda" como referência ao impacto da pandemia de Covid-19 é representativa das formas como o conhecimento tem circulado nas sociedades contemporâneas. A expressão nasceu de uma postagem na rede social Twitter, por parte de um pneumologista estadunidense, em 30 de março de 2020 (TSENG, 2020). Na postagem, há um gráfico onde se apontam as consequências da Covid-19 em quatro ondas: a primeira correspondendo à mortalidade e morbidade imediatas pela Covid-19; a segunda, ao impacto da restrição de recursos para pacientes com outros problemas de saúde; a terceira, às consequências da interrupção no cuidado de pacientes com doenças crônicas; e, finalmente, a quarta, que incluiria trauma psíquico, doença mental, impacto econômico e burnout.

Observa-se que, em sua origem, o modelo proposto não é medicalizante. $\mathrm{O}$ autor chega a comentar, na mesma postagem, que entende o "trauma psíquico" como psicossocial e o define como fruto dos "efeitos cumulativos do desamparo e do medo" gerados pela pandemia (TSENG, 2020). O modelo e o gráfico original, ou de forma adaptada, passaram a ser reproduzidos em periódicos científicos, como, por exemplo, em O'Connor et al. (2021), ainda se referindo às consequências da Covid-19 no campo da saúde mental sem reduzi-las a um espectro de distúrbios mentais. No caso brasileiro, porém, a expressão passou a ser ressignificada a partir de um viés claramente medicalizante.

\section{A "quarta onda" como estratégia discursiva do poder psiquiátrico no Brasil}

A $A B P$ vem se apresentando no cenário pandêmico brasileiro como grande apoiadora e divulgadora da expressão "quarta onda" da Covid19, ou, como é chamada por ela: "a onda das doenças mentais". Esta expressão surgiu nas narrativas midiáticas da $\mathrm{ABP}$ menos de dois meses depois de sua criação. Em 21 de maio de 2020, o psiquiatra Antônio Geraldo da Silva (atual presidente da ABP e da Associação Psiquiátrica da América Latina) afirmou, em um debate online promovido pela $A B P$, que "enquanto as curvas [dos infectados pelo coronavírus] diminuem no mundo, as consequências relacionadas à saúde mental não somente são maiores, como durarão mais tempo". Nesta mesma live, intitulada Covid-19 e saúde mental: o que é a quarta onda?"2 (a partir de agora citada como "live 1"), afirmou-

\footnotetext{
${ }^{2}$ Nesta live (COVID-19..., 2020), transmitida pela Universidade Fundação Mineira de Educação e Cultura (Fumec), ao lado de Antônio Geraldo Silva, participou do debate o psicólogo e professor universitário Leandro F. Malloy-Diniz, presidente da Associação Brasileira de Impulsividade e Patologia Dual (Abipad).
} 
se ainda a presença de um diálogo em curso entre $A B P$, governadores e poder público sobre a saúde mental no contexto da pandemia.

Antes do uso da expressão "quarta onda", a ABP já divulgava em suas redes sociais (Facebook e Instagram) materiais sobre a saúde mental desde a chegada da pandemia da Covid-19 ao Brasil, com informativos, debates virtuais sobre populações específicas nesse contexto e com uma campanha de perspectiva individualizante para orientação e incentivo ao home office, com foco acrítico na produtividade laboral.

A partir da utilização da modelagem matemática como ferramenta para prever padrões hipotéticos da "história natural da doença" (SILVA et al., 2020) e dos resultados de uma pesquisa não publicada - realizada pela própria $\mathrm{ABP}$ com seus associados e que apontaria um "aumento na clientela de psiquiatras" no Brasil) - Silva et al. (2020) concluíram que há a necessidade de organização de uma política pública baseada em evidências para o enfrentamento da "quarta onda" (live 1). No debate, Malloy-Diniz comentou que "não dá para fazer política pública por tentativa e erro, não é festa", e foi complementado por Silva: "nem em ideologia" (live 1).

Partindo destas afirmações, questiona-se: considerando todo o complexo aspecto moral envolvido na história da loucura e da normalidade, é possível a produção de um saber sobre o existir humano que não seja ideológico? O mito da neutralidade científica, há anos questionado especialmente no campo da saúde mental, se evidencia aqui em primeiro plano. A própria escolha do conceito "doença mental" para se referir às vivências de sujeitos em sofrimento mental é, afinal, ideológica, promovendo o foco de atenção às doenças e não às pessoas (AMARANTE, 2007). Nesta direção, o presidente da ABP é categórico em sua intervenção no debate em questão, dizendo que não devemos confundir as "doenças mentais" "com o nome que as pessoas costumam usar por aí, de sofrimento psíquico. [...] O que nós estamos falando aqui é de doença: tem conjunto de sinais e sintomas" (live 1).

A postura medicalizante é ressaltada em uma transmissão posterior sobre o mesmo tema ${ }^{3}$ (a partir daqui citada como live 2), em que Silva diz que o que tem gerado o aumento de "doenças mentais" no contexto pandêmico são determinantes sociais tais como a pobreza, o desemprego e a migração. De fato, é notório que o aumento da fome e do desemprego no Brasil e que outras desigualdades e precariedades tais como o racismo estrutural e as hierarquias de gênero são fatores vulnerabilizantes nos desdobramentos concretos da pandemia de Covid-19 (BIRMAN, 2020). Contudo, circunscrever o sofrimento mental nesse contexto à esfera exclusiva da "doença mental" é um modo flagrante de medicalização da vida.

\footnotetext{
${ }^{3}$ Intitulada Quarta onda - a onda das doenças da mente e Covid-19, como fazer?, com a presença de Antônio Geraldo Silva e dos debatedores Múcio Bonifácio Guimarães, Ademir Cândido da Silva, Edilberto Pereira da Silva e Arlindo Chapetta (QUARTA ONDA..., 2020). Esta transmissão ao vivo foi realizada e transmitida pela instituição maçônica Grande Oriente do Brasil (GOB).
} 
No contexto histórico-social neoliberal em que nos encontramos, atitudes individuais têm sido valorizadas em detrimento de ações coletivas e estatais para o enfrentamento da pandemia. Na polarização que se estabeleceu entre um imperativo da economia e um imperativo da vida, o governo brasileiro opta pelo imperativo econômico de maneira escatológica em seus discursos negacionistas (BIRMAN, 2020). Nesta conjuntura, é possível pensar (ainda) em um padrão funcionalista de normalidade como um crivo para "doenças mentais"? Aparentemente, isto é o que faz o discurso do representante maior da $\mathrm{ABP}$, ao anunciar que os sintomas de medo do coronavírus e de sair de casa passam a ser uma doença no momento em que te "geram prejuízos" e "atrapalham a produção/trabalho" (live 2). Em suas palavras: "Temos catalogadas mais de seiscentas doenças [mentais] [...], saiu do padrão de funcionamento, liga o alerta" (live 2).

Uma perspectiva medicalizante também é acionada quando o debate sobre o suicídio na pandemia é levantado nas transmissões. Nelas, uma "epidemia de suicídios" é prevista para o Brasil como consequência da "epidemia de doenças mentais", considerando-se, nas palavras de Silva (live 2), que "cem por cento daqueles que se suicidam é por causa de doenças mentais" e que o aumento do suicídio no Brasil se dá porque "O sistema público de saúde está falido".

Analisar as condições de enunciação de determinadas práticas discursivas tem se mostrado uma importante ferramenta no campo da saúde mental (AMARANTE, 2007). No esteio desta constatação, quando a ABP afirma uma hipotética epidemia de suicídios devido à falência do sistema público de saúde brasileiro, não se pode deixar de considerar que tal discurso ecoa uma série de outros pronunciamentos em que seu presidente critica a reforma psiquiátrica ocorrida no Brasil.

Nas palavras de Silva (live 1): "Nos últimos trinta anos houve uma diminuição de mais de cem mil leitos psiquiátricos do SUS. O que foi feito? Pegou-se mais de cem mil pessoas e jogou-se nas ruas. Jogaram nas ruas sem ter nada para poder acolher, substituir, para cuidar dessas pessoas". Além disso, cabe ressaltar que, em artigo publicado na Veja Saúde, Silva (2020) considera que: "Ao chegar ao Brasil, a pandemia se soma a um cenário de desassistência pública em saúde mental, tornando a busca por soluções ainda mais árdua após 30 anos de políticas inadequadas nesse contexto". Para ele, "os avanços em psiquiatria aplicados com sucesso no serviço privado não se comparam ao que é disponibilizado no atendimento público, configurando um obstáculo para o acompanhamento médico e a oferta de tratamento eficaz e equânime a boa parte da nossa população".

Neste momento de nossa análise, podemos relacionar os discursos do governo federal e da ABP considerando a parceria pública entre esta instituição e o Ministério da Saúde (MS) para enfrentamento da "quarta onda" da Covid-19 no Brasil, que tem como um de seus elementos a confec- 
ção de uma cartilha desenvolvida por ambos ${ }^{4}$. Na live 2, esta parceria é mencionada mais de uma vez e a interlocutora anunciada como referência das ações da ABP no MS é a médica Mayra Pinheiro, secretária nacional de gestão do Trabalho e da Educação em Saúde. Por ser defensora de medicamentos ineficazes para o tratamento da Covid-19 (tais como a cloroquina e a hidroxicloroquina), Mayra Pinheiro também é popularmente conhecida como "Capitã Cloroquina" . Nesta trama, a proposta de considerar categoricamente o sofrimento mental decorrente dos desdobramentos da situação endêmica que assola o país como associado a "doenças mentais" parece indicar um interesse comum de ambas as instituições analisadas, que se consolida posteriormente: a compra de medicamentos como resposta à crise sanitária decorrente da pandemia.

\section{Medicalizando o "impacto social" da pandemia: o avanço sobre as políticas públicas}

A promessa de investimentos públicos para a inserção de "medicamentos para tratamentos psiquiátricos dentro da cesta básica que está fornecendo para questão do tratamento da Covid", anunciada por Silva na live 2, se consolida em setembro de 2020 com a liberação orçamentária de $\mathrm{R} \$$ 649.833.472,83 para a compra de psicotrópicos por parte do Ministério da Saúde. ${ }^{6}$ Em 22 de setembro de 2020 foi publicada a Portaria 2.516, que "dispõe sobre a transferência de recursos financeiros de custeio para a aquisição de medicamentos do Componente Básico da Assistência Farmacêutica utilizados no âmbito da saúde mental em virtude dos impactos sociais ocasionados pela pandemia da COVID-19", no ato do gabinete do então ministro da saúde Eduardo Pazuello (BRASIL, 2020b). A linha argumentativa presente no documento oficial do Ministério da Saúde exemplifica o processo de medicalização presente: embora haja o reconhecimento na portaria de que há "impactos sociais" da pandemia, a transferência de recursos públicos para a compra de psicotrópicos é proposta como solução, em um reducionismo extremo das complexas consequências sociais da pandemia a algo de ordem puramente biomédica.

A lista presente no Anexo I da portaria citada (BRASIL, 2020b) traz quatro anticonvulsivantes, quatro estabilizadores de humor e antidepressivos, quatro antipsicóticos, quatro antiparksonianos, três ansiolíticos, além de um anti-histamínico, um hipnossedativo e um suplemento vitamínico. Assim, esta transferência de recursos para os medicamentos listados não foi justificada pela utilização consoante com a Lei $n^{\circ} 10.2016$, de 6 de

\footnotetext{
${ }^{4}$ A cartilha intitulada Saúde mental e Covid-19 foi lançada em junho de 2020 a partir de uma parceria entre essas duas instituições (BRASIL, 2020a).

${ }^{5}$ Sobre a vida social da cloroquina no contexto da medicalização da política brasileira, ver Gonçalves (2020).

${ }^{6}$ Neste ponto, a questão filosófica de Michel Foucault (2006, p. 75) ainda nos parece muito relevante: "¿cómo un saber tan parco puede arrastrar tanto poder?".
} 
abril de 2001, que, em seu Art. $2^{\circ}$, sobre os atendimentos em saúde mental, afirma ser de direito cientificar a pessoa portadora de transtorno mental e seus familiares de tudo o que Ihes protege, a exemplo da alínea VII - "ser tratada em ambiente terapêutico pelos meios menos invasivos possíveis" (BRASIL, 2001). O endosso ministerial para a compra de psicotrópicos em larga escala se deu tão somente com a justificativa de uso voltado para os impactos sociais decorrentes da pandemia da Covid-19.

O discurso da "quarta onda" também é reproduzido pelo Ministério de Saúde, que publicou em sua página, em 22 de fevereiro de 2021, uma notícia tratando dos aspectos da saúde mental consequentes da pandemia (BRASIL, 2021). Nesta, há um alerta para a "quarta onda" e para os comportamentos e reações comuns que a população poderia ter ao longo da pandemia. Chama a atenção que estes aspectos, bem como as orientações que o Ministério da Saúde divulgou para a população, foram retirados do Guia de Saúde Mental Pós-Pandemia (PFIZER, 2020), documento construído em uma iniciativa entre o Instituto de Ciências Integradas (INI/CCM Group) e a Upjohn, naquela época uma divisão da Pfizer e atualmente parte do grupo Viatris (PFIZER, 2021). O guia foi desenvolvido pela Pfizer como um documento de referência para médicos e pacientes, abordando temas como impactos na saúde mental de crianças e adolescentes, idosos, transtornos psiquiátricos, aumento da ansiedade na população, saúde mental dos profissionais, telemedicina, orientações individuais para que o público procure acesso a serviços de saúde e estratégias pessoais para lidar com os sintomas mais comuns de acordo com faixa etária ou sintomas vividos.

Ainda com relação ao uso de psicotrópicos no Brasil durante a pandemia de Covid-19 no Brasil, o Conselho Federal de Farmácia noticiou, em setembro de 2020, que uma consultoria realizada pela IQVA mostrou um aumento significativo deste uso (CONSELHO FEDERAL DE FARMÁCIA, 2020). O aumento, comparado ao ano anterior (2019), seria de $14 \%$ na venda de antidepressivos e estabilizadores de humor e de $13 \%$ na venda de anticonvulsivantes. Esse aumento se daria pelas consequências da suposta "quarta onda". Ainda na mesma notícia, o CFF aponta o principal meio através do qual acontecem tentativas de suicídio no país, por intoxicação medicamentosa, um dado relevante ao considerar este aumento na venda e consumo de psicotrópicos.

Políticas públicas direcionadas ao uso de psicotrópicos como "cha$v^{\prime \prime}$ para o sofrimento mental se relacionam diretamente à minimização da necessidade de suporte psicossocial para as pessoas que têm necessidade de apoio nesse quesito. Observa-se que, nos discursos da ABP e do MS, o fortalecimento da Rede de Atenção Psicossocial, construída a partir de um processo histórico durante décadas, fica em segundo plano, deixando de ser priorizado por parte das políticas públicas no campo. Em termos comparativos, a suplementação orçamentária para a Rede de Atenção 
Psicossocial, justificada a partir dos efeitos da Covid-19 sobre a saúde mental, no valor total de aproximadamente $\mathrm{R} \$ 165$ milhões, destinados por meio das portarias 2.972, 2.973, 2.974, 2.975, 2.976, 2.977, 2.982, 2.983, 2.985 e 2.987 do MS (Brasil, 2020c) e da Portaria GM/MS no 3.350 (BRASIL, 2020d), é mais de quatro vezes menor do que a destinada à compra de psicotrópicos, o que mostra a inversão da prioridade dada durante a reforma psiquiátrica brasileira ao desenvolvimento dessa rede.

\section{Considerações finais ou "por uma vida fora das caixas"}

O viés medicalizante na abordagem do sofrimento mental tem sido objeto de estudos e vem sendo combatido por movimentos alinhados com a reforma psiquiátrica, a luta antimanicomial, a defesa do SUS e das políticas públicas de saúde mental, no âmbito acadêmico e nas práticas de resistência da sociedade civil organizada, que reivindica garantias para o respeito ao cuidado integral em liberdade. Na ofensiva da contrarreforma psiquiátrica brasileira e seus ataques aos defensores dos direitos humanos no campo da saúde mental, os agentes da contrarreforma têm se apoiado, durante a pandemia da Covid-19, no reducionismo biomédico e na medicalização do sofrimento mental, por meio da divulgação do discurso de "quarta onda", que associa seu aumento presumido a uma suposta "epidemia de transtornos mentais".

Em um contexto internacional, a crítica ao foco individualizante e medicalizante no campo da saúde mental tem conquistado espaço crescente. Pûras (2019) apontou a necessidade de intervenções estruturais para a diminuição das diferenças e desigualdades que produzem sofrimento mental, com ênfase nas abordagens baseadas em direitos humanos. O documento, apresentado ao Conselho de Direitos Humanos da ONU, cita como determinantes políticos e sociais do sofrimento mental a xenofobia, dentre outras formas de discriminação resultantes de injustiças históricas, legados do colonialismo e da escravidão, que produzem trauma social por gerações. Reduzir desigualdades e sistemáticas assimetrias de poder e exclusão social como a que afeta mulheres e LGBTs, ou a negligência do direito das crianças e jovens, é uma condição para produzir relações não violentas e respeitosas que apoiam a saúde mental (PÛRAS, 2019).

Com relação aos efeitos na saúde mental da pandemia de Covid19 no Brasil, concordamos com os apontamentos de Caponi (2020), para quem, em contextos de desamparo e temor frente a uma ameaça externa, as alterações de sono, apetite, sensação de culpa ou inutilidade podem ser "perfeitamente normais", assim como sentir raiva e impotência em uma pandemia e ter medo da morte diante de uma tragédia coletiva anunciada perante um Estado que abandonou os cidadãos, principalmente as comunidades pobres, os negros e os indígenas. 
Ainda que medicamentos psicotrópicos possam ser adjuvantes no tratamento de vários quadros relacionados ao sofrimento mental, a centralidade de seu uso como "solução", em detrimento da atenção psicossocial necessária para acolhimento de pessoas com este sofrimento, configura-se como uma estratégia discursiva de cunho medicalizante. Se, como vimos anteriormente, há diversas pesquisas dissonantes em relação ao suposto aumento de "distúrbios psiquiátricos" durante a pandemia, desenvolvidas inclusive dentro de um paradigma biomédico, o aumento de uso de medicamentos psicotrópicos nesse período no Brasil pode ser visto como um avanço do processo de medicalização da vida em nossa sociedade.

Finalmente, é importante considerar que a construção discursiva de uma suposta "quarta onda" da Covid-19 por parte dos segmentos conservadores da psiquiatria brasileira, e sua consequente influência sobre as políticas públicas a serviço da lógica individualizante e medicalizante, não pode ser entendida como um projeto apenas de uma determinada corporação - no caso, de psiquiatras. A medicalização do sofrimento mental corresponde a um apagamento das diversas formas de opressão que o geram, bem como à deslegitimação dos movimentos sociais que vêm apontando de forma crescente as consequências traumáticas das violências perpetradas nas várias esferas sobre as subjetividades, no Brasil e em outros países. O posicionamento crítico diante deste processo é um elemento essencial para a defesa de uma concepção de saúde mental democrática, pautada nos direitos humanos e nos princípios da reforma psiquiátrica brasileira. 


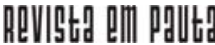

\} CONTRARREFORMA PSIQUIÁTRICA BRASILEIRA - GARCIA, M. R. V.; AMORIM, S. C.; RODRIGUES, G. V.; MENDONÇA, L. H. F. \} DOI: $10.12957 /$ REP.2022.63525

\section{Referências}

AMARANTE, P. Saúde mental e atenção psicossocial. Rio de Janeiro: Editora Fiocruz, 2007.

AMARANTE, P; NUNES, M. de O. A reforma psiquiátrica no SUS e a luta por uma sociedade sem manicômios. Ciência e Saúde Coletiva, v. 23, n. 6, 2018.

ASSOCIAÇÃO BRASILEIRA DE PSIQUIATRIA. Ofício n. 109/20. Necessidade de inclusão de importantes psicotrópicos na lista da Rename - Errata. Rio de Janeiro, 2 abr. 2020. Disponível em: https://d494f813-3c95-463a898c-ea1519530871.filesusr.com/ugd/c37608_28e221a504d44be091aa7 78d4d0cbe91.pdf. Acesso em: 24 jun. 2021.

BIRMAN, J. O trauma na pandemia do coronavírus: suas dimensões políticas, sociais, econômicas, ecológicas, culturais, éticas e científicas. 1a ed. Rio de Janeiro: Civilização Brasileira, 2020.

BRASIL. Ministério da Saúde. Lei n. 10.216, de 06 de abril de 2001. Disponível em: planalto.gov.br/ccivil_03/leis/leis_2001/l10216.htm. Acesso em: 3 maio. 2021.

BRASIL. Ministério da Saúde. Saúde Mental e Covid-19. Cartilha produzida em parceria com a Associação Brasileira de Psiquiatria. Brasília, 2020a. Disponível em:

https://www.gov.br/saude/pt-br/centrais-de-conteudo/manual-sa-de-mentale-covid-19-pdf. Acesso em: 24 jun. 2021.

BRASIL. Ministério da Saúde. Portaria n. 2.516, de 21 de setembro de 2020, 2020b. Disponível em: https://brasilsus.com.br/index.php/pdf/portaria-no2-516/. Acesso em: 24 jun. 2021.

BRASIL. Ministério da Saúde. Ministério da Saúde investe mais de $R$ \$ 65 milhões na ampliação da rede de saúde mental, 2020c. Disponível em https://aps.saude.gov.br/noticia/10217. Acesso em: 24 jun. 2021.

BRASIL. Ministério da Saúde. Portaria GM/MS no 3.350, de 8 de dezembro de 2020, 2020d. Disponível em: https://brasilsus.com.br/wp-content/ uploads/2020/12/portaria3350.pdf. Acesso em: 24 jun. 2021.

BRASIL. Ministério da Saúde. Saúde mental e a pandemia de Covid-19. Brasília, 2021. Disponível em: https://bvsms.saude.gov.br/saude-mental-ea-pandemia-de-covid-19/. Acesso em: 24 jun. 2021.

BRUNONI, A. R. et al. Prevalence and risk factors of psychiatric symptoms and diagnoses before and during the Covid-19 pandemic: findings from the Elsa-Brasil Covid-19 mental health cohort. Psychological Medicine, 2021.

CAPONI, S. N. C. de. Necropolítica e sofrimento psíquico em tempos de pandemia. Mad in Brasil: ciência, psiquiatria e justiça social, Rio de Janeiro, 
18 ago. 2020. Disponível em: https://madinbrasil.org/2020/08/necropoliticae-sofrimento-psiquico-em-tempos-de-pandemia. Acesso em: 24 jun. 2021. CONSELHO FEDERAL DE FARMÁCIA. Venda de medicamentos psiquiátricos cresce na pandemia. Brasília, 10 set. 2020. Disponível em: https://www. cff.org.br/noticia.php?id=6015\&titulo $=$ Venda + de+medicamentos + psiqui\%C3\%A1 tricos+cresce+na+pandemia. Acesso em: 24 jun. 2021.

COVID-19 e a saúde mental: o que é a quarta onda? [Palestra de]: Antônio Geraldo da Silva e Leandro F. Malloy-Diniz. [S. I.]: Canal Universidade Fumec, 21 maio 2020. Transmissão ao vivo. Disponível em: https://www .youtube.com/watch?v=kfrukjlvwUY. Acesso em: 24 jun. 2021.

DUARTE, M. J. de O. et al. Pandemia, saúde mental e drogas: as vidas precárias como questão no serviço social. In: GOMES, T. M. da S.; PASSOS, R. G.; DUARTE, M. J. de O. (Org.). Saúde mental e drogas em tempos de pandemia: contribuições do Serviço Social. Uberlândia: Navegando Publicações, 2020.

FANCOURT, D.; STEPTOE, A.; BU, F. Trajectories of anxiety and depressive symptoms during enforced isolation due to Covid-19 in England: a longitudinal observational study. The Lancet Psychiatry, v. 8, n. 2, 2021.

FOUCAULT, M. Soy un artificiero. In: ROIT, R.; RIUS, R.; SALVAT, P. (Org.). Entrevistas con Michel Foucault. Barcelona: Paidós, 2006.

FOUCAULT, M. Os anormais: curso no Collège de France (1974-1975). São Paulo: Martins fontes, 2010.

GARCIA, M. R. V. Políticas de prevenção ao suicídio no Brasil e seu impacto sobre as escolas. Práxis Educacional, v. 15, n. 36, 2019.

GONÇALVES, F. R. Medicalização da política: a vida social da cloroquina e seus demais agenciamentos. Ponto Urbe, n. 27, 2020.

LOBOSQUE, A. M. Foucault e a luta antimanicomial brasileira: uma intensa presença. Psicologia em Revista, Belo Horizonte, v. 24, n. 1, abr. 2018.

MORGAN, C.; ROSE, N. Multidisciplinary research priorities for the Covid19 pandemic. The Lancet Psychiatry, v. 7, n. 7, 2020.

O'CONNOR, K. et al. Mental health impacts of COVID-19 in Ireland and the need for a secondary care mental health service response. Irish journal of psychological medicine, v. 38, n. 2, 2021.

PAN, K. Y. et al. The mental health impact of the COVID-19 pandemic on people with and without depressive, anxiety, or obsessive-compulsive disorders: a longitudinal study of three Dutch case-control cohorts. Lancet Psychiatry,v. 8, n, 2, 2021.

PASSOS, R. G. Holocausto ou navio negreiro?: inquietações para a reforma psiquiátrica brasileira. Argumentum, Vitória, v. 10, n. 3, set./dez. 2018. 


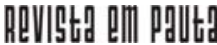

\} CONTRARREFORMA PSIQUIÁTRICA BRASILEIRA - GARCIA, M. R. V.; AMORIM, S. C.; RODRIGUES, G. V.; MENDONÇA, L. H. F. \} DOI: $10.12957 /$ REP.2022.63525

PFIZER. U. Guia de saúde mental pós-pandemia no Brasil. 2020. Disponível em: https://www.pfizer.com.br/sites/default/files/inline-files/Guia-de-SaudeMenta-\%20pos-pandemia-Pfizer-Upjohn.pdf. Acesso em: 24 jun. 2021.

PFIZER. Lançamento da Viatris, nova empresa global de cuidados de saúde, resultado da fusão da empresa Mylan com a Upjohn, uma divisão Pfizer. São Paulo, 1 jun. 2021. Disponível em: https://www.pfizer.com.br/lan camento-da-viatris-nova-empresa-global-de-cuidados-de-saude-resultadoda-fusao-da-empresa-mylan. Acesso em: 24 jun. 2021.

PÛRAS, D. Report of the special papporteur on the right of everyone to the enjoyment of the highest attainable standard of physical and mental health. 2019. Disponível em: https://digitallibrary.un.org/record/3803412. Acesso em: 24 jun. 2021.

QUARTA ONDA: a onda das doenças da mente e Covid-19, como fazer? [Palestra de]: Antônio Geraldo da Silva. [S. I.]: Canal TV GOB, 19 out. 2020. Transmissão ao vivo. Disponível em: https://www.youtube.com/ watch?v=reLAGMtH-5U. Acesso em: 24 jun. 2021.

RUSSO, J. Do desvio ao transtorno: a medicalização da sexualidade na psiquiatria contemporânea. In: CARRARA, S.; GREGORI, M. F.; PISCITELLI, A. Sexualidades e saberes: convenções e fronteiras. Rio de Janeiro: Garamond, 2004.

SILVA, A. G. da. Uma onda de doenças mentais vem com a Covid-19. Precisamos agir já! Blog Com a Palavra - Veja Saúde, São Paulo, 19 ago. 2020. Disponível em: https://saude.abril.com.br/blog/com-a-palavra/umaonda-de-doencas-mentais-vem-com-a-covid-19-precisamos-agir-ja/. Acesso em: 24 jun. 2021.

SILVA, A. G. da et al. Mental health: why it still matters in the midst of a pandemic. Brazilian Journal of Psychiatry, v. 42, n. 3, 2020.

TSENG, V. As our friends and colleagues brave the font lines [...]. Atlanta, 30 mar. 2020, 14:04. Twitter: @VectorSting. Disponível em https://twitter. com/VectorSting/status/1244671755781898241. Acesso em: 24 jun.2021.

DOI: $10.12957 /$ rep.2022.63525

Recebido em 24 de junho de 2021.

Aprovado para publicação em 06 de outubro de 2021.

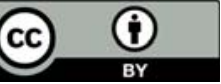

A Revista Em Pauta: Teoria Social e Realidade Contemporânea está licenciada com uma Licença Creative Commons Atribuição 4.0 Internacional. 